\title{
El Método Científico como Alternativa Didáctica de Educación en Valores para Escuelas de Ingeniería
}

\author{
Gloria M. Jaime-Mirabal ${ }^{1}$ y Delfino Ladino-Luna ${ }^{2^{*}}$ \\ ${ }_{1}$ CES, Universidad de Pinar del Río, Pinar del Río, Cuba (e-mail: gjaimem@upr.edu.cu) \\ 2 Física de Procesos Irreversibles, C. B., Universidad Autónoma Metropolitana-A, Cd. de México, México \\ (e-mail: dll@correo.azc.uam.mx)
}

*Autor a quien debe ser dirigida la correspondencia

Recibido Ene. 22, 2018; Aceptado Mar. 28, 2018; Versión final May. 30, 2018, Publicado Oct. 2018

\begin{abstract}
Resumen
En este trabajo se discuten las principales ideas de la Educación en Valores, y la manera como aparece implícitamente cuando se enseña como se proponen y prueban las teorías sobre el comportamiento de la naturaleza. Primero se exponen los principios y objetivos de la Educación en Valores. Luego se muestran los principios básicos del Método Científico. Finalmente se observa cómo al poner en práctica el Método Científico, se aplican implícitamente los principios de la Educación en Valores. Para hacer explícita esta idea se utilizan diagramas de flujo, mostrando la influencia de las decisiones tomadas a partir de una hipótesis (Método Científico), y su relación con los principios básicos de la Educación en Valores. Así, se busca una posible relación biunívoca entre la Teoría de la Educación en Valores y el Método Científico, que permita utilizar este método para fortalecer la apropiación de valores en los estudiantes.
\end{abstract}

Palabras clave: educación en valores; método científico; formación socio-humanista; docencia; metodología educativa

\section{The Scientific Method as a Didactic Alternative of Values Education for Engineering Schools}

\begin{abstract}
In this work the main ideas of Values Education are discussed, and the implicit way in which they appear when it is taught how theories about the behavior of nature are proposed and tested. First, the principles and objectives of Values Education are exposed. Then, the basic principles of the Scientific Method are shown. Finally, it is observed that when implementing the Scientific Method, the principles of Values Education are implicitly applied. To make this idea more explicit, flowcharts are used, showing the influence of the decisions taken from a hypothesis (Scientific Method), and its relation with the basic principles of Values Education. Thus, a possible bi-univocal relationship between the Theory of Values Education and the Scientific Method is sought, which allows us to use this method to strengthen the appropriation of values in students.
\end{abstract}

Keywords: values education; scientific method; socio-humanist formation; teaching; educational methodology 


\section{INTRODUCCIÓN}

Generalmente se piensa que las teorías de la educación tienen su principal campo de aplicación en los niveles básicos de la enseñanza: los primeros nueve años de escuela, después del pre-escolar (llámese Kindergarden, pre-primaria o cualquier otro nombre). Sin embargo, si uno revisa la llamada Teoría de la Educación en Valores (Arana y Batista, 1997; Barba, 2005; Batista y Reyes, 1997; Garcell, 2010; Şahinkayasi y Kelleci, 2013; Schmelkes, 1996), encuentra que existe una relación entre ésta y el llamado Método Científico con el cual se enseñan, a nivel universitario, los fundamentos de las llamadas ciencias duras (química, física, biología, entre otras semejantes). La primera aspira a lograr en el estudiante una estructuración de su pensamiento para obtener un desempeño profesional íntegro; el segundo aspira a que en el desarrollo del pensamiento prevalezca el apego a la libre discusión de las teorías, sin trasgredir los principios básicos de cada ciencia, en palabras llanas pudiera expresarse, sin hacer trampa. Esto es, tomando como principio una hipótesis, de manera que dentro del marco de ésta se encuentren resultados que reflejen el actuar de la naturaleza, hasta donde es posible (Einstein, 1934; Poincaré, 1981; Popper, 2005), y tomando en cuenta que "el aprendizaje de cualquier disciplina debe contar con el espíritu con que el estudiante se aproxime a ésta" (Córdoba y Rojo, 1985), esencialmente sin temor, con interés y la idea de aprovechar para la vida futura lo que pueda aprender.

En la práctica del proceso de enseñanza-aprendizaje existe actualmente la idea de que es necesario desarrollar valores en el estudiante, a través de la ampliación del nivel cultural general, al mismo tiempo que los métodos de adquirir conocimiento y técnicas de investigación, desarrollando estilos de pensamiento y habilidades de comunicación (Baños-Martínez, 2013; Castiglione, 2014; Thornberg, 2008). Para arribar al objetivo de que el estudiante se convierta en un individuo productivo y comprometido con la sociedad en donde se desarrollará como adulto, una tendencia significativa en los últimos años, no siempre lograda, ha sido la de dar relevancia al componente humanista mediante las ciencias sociales en la formación de ingenieros, pues se espera que de esa manera el futuro profesional ejerza con calidad su profesión. Se espera que estas disciplinas favorezcan el desarrollo de una formación más completa en el futuro ingeniero y de esa manera ejerza con calidad su profesión.

Como consecuencia de la idea previa aparece la polémica sobre la manera de lograr el objetivo planteado, a saber, si la formación socio-humanista se alcanza mediante la adición de asignaturas de ciencias sociales, o si los conocimientos obtenidos de las ciencias sociales deben insertarse dentro de las disciplinas propias de la ingeniería. La experiencia ha mostrado que una adecuada alternativa consiste en integrar simbióticamente las posibilidades que brindan los contenidos de las ciencias sociales, con las ciencias naturales y exactas desde un enfoque interdisciplinario (Berkowitz, 2011; Carr, 2011; Halstead y Taylor, 2000; Lovat, et al, 2011; Sheldrake, et al, 2017). Una herramienta básica para desarrollar esta alternativa es el diseño de programas de Tecnología Apropiada, que consiste en "mostrar con sencillez y sensibilidad la tecnología en beneficio del hombre y su contexto, al mismo tiempo que se plantea un cambio de valores en la ingeniería y la enseñanza de los elementos que la componen, revitalizando los valores que se piensa que debe poseer todo ingeniero en su profesión" (Arana y Armenteros 1996).

En el contexto de los párrafos anteriores, en el presente trabajo se discute la manera como la teoría de la Educación en Valores está implícita al momento de desarrollar las ideas básicas del llamado Método Científico y, a su vez, cómo el Método Científico ha sido usado de manera implícita al momento de plantear una alternativa de desarrollo de los valores en el futuro ingeniero. Para aclarar mejor este planteamiento, el trabajo se desarrolla de la siguiente manera: primero de forma breve se plantean los principios básicos de la Educación en Valores, a continuación se discuten brevemente las ideas básicas del Método Científico, y finalmente se muestra como aparecen las ideas de uno en el otro y viceversa, de manera que se pueda concluir que al momento de enseñar las ciencias duras necesarias en la ingeniería, se está desarrollando una Educación en Valores. De esta forma se muestran, a través de esquemas gráficos, las relaciones entre las llamadas dimensiones necesarias y un conjunto de valores básicos elegidos para la profesión, también se discute la manera cómo al aplicar ciertos aspectos del Método Científico para la adquisición de conocimiento útil para el futuro ingeniero, al mismo tiempo se está reforzando la adquisición de dicho conjunto de valores.

\section{LA EDUCACIÓN EN VALORES, UNA ALTERNATIVA METODOLÓGICA}

Toda teoría, dentro de cualquier rama del conocimiento humano, parte de un objetivo propuesto por la necesidad de obtener un resultado que contenga las aspiraciones de la sociedad donde se desarrolla. Es el caso de la preparación de profesionales, y en particular de profesionales en ingeniería (en cualquiera de sus especialidades). La ingeniería en general se define como la actividad que aplica un conjunto de conocimientos científicos y tecnológicos para la innovación, invención, desarrollo y mejora de técnicas y herramientas para satisfacer las necesidades del entorno en que vive: la sociedad (Grech, 2001). El ingeniero utiliza los conocimientos de las llamadas ciencias duras, tanto para el desarrollo de tecnologías, 
como para el manejo eficiente y productivo de recursos y fuerzas de la naturaleza en beneficio de la sociedad. En este sentido la ingeniería es una actividad que transforma el conocimiento en algo práctico.

Se dice comúnmente que el ingeniero debe tener una vocación de servicio. Sin embargo, las diferentes situaciones que ha vivido y vive actualmente, cada individuo, no necesariamente permiten que se obtenga esa vocación de servicio. Una alternativa de infundir dicha vocación es a través del desarrollo de teorías pedagógicas que se puedan llevar a la práctica en el nivel propiamente de desarrollo del futuro ingeniero, durante su formación en la enseñanza superior. Es así que se ha desarrollado una interesante teoría pedagógica llamada Educación en Valores. La educación moral, al igual que las normas socio-jurídicas, tienen el objetivo de colaborar para lograr un comportamiento ligado a los valores responsabilidad y ética, que se aspira fomentar desde la niñez. No obstante en la actualidad parece que se vive en una sociedad sin valores; o en el mejor de los casos, han aparecido nuevos valores asociados al nuevo paradigma socioeconómico y cultural derivado de la globalización que ha traído consigo el desarrollo de las comunicaciones. También hay quien piensa que el problema está en la existencia de una multi-variedad de valores, lo que produce confusión y desorientación en la actuación y valoración de los seres humanos. Quizás esté ocurriendo todo ello. Ante este reto la teoría pedagógica de la Educación en Valores, puede contribuir a la formación de los estudiantes durante el desarrollo de las tareas docentes mediante el trabajo en grupos, fomentándose principios morales que regulen su comportamiento, con el objetivo de lograr una mejor convivencia. Las técnicas de discusión, intercambio y reflexión de tópicos actuales, podría ser una de las vías útiles para evaluar las opiniones y contrastar sus puntos de vista.

De acuerdo al marco teórico descrito, la Educación en Valores se centra en establecer una perspectiva referida a la acción pedagógica. Un ambiente pedagógico donde el aprendizaje vaya a las raíces mismas del comportamiento del alumno, ya que de lo que se trata es de: educar y formar, no sólo instruir, reconocer que el crecimiento de una persona autónoma y responsable en el plano moral es prioritario, lograr que la formación del carácter preceda a la instrucción, ayudarles a superar sus problemas afectivos, sostenerlos en la resolución de sus problemas familiares y sociales, acompañarles en la larga conquista de la autonomía y responsabilidad en estos tiempos de crisis de la evolución humana. Esta acción pedagógica no se concibe como una negación de los valores como objetivos o ideales, más bien se centra en la premisa de que la misma persona va auto-construyendo su aprendizaje a través de la interacción social donde asimila valores, desecha anti-valores y reorganiza en forma jerárquica su propia normativa o escala de valores (BañosMartínez, 2013; Castiglione, 2014; Hoyos et al., 2001; Simmons, 2005; Thornberg, 2008). En este sentido el proceso de enseñanza-aprendizaje puede plantearse como se muestra en la Figura 1.

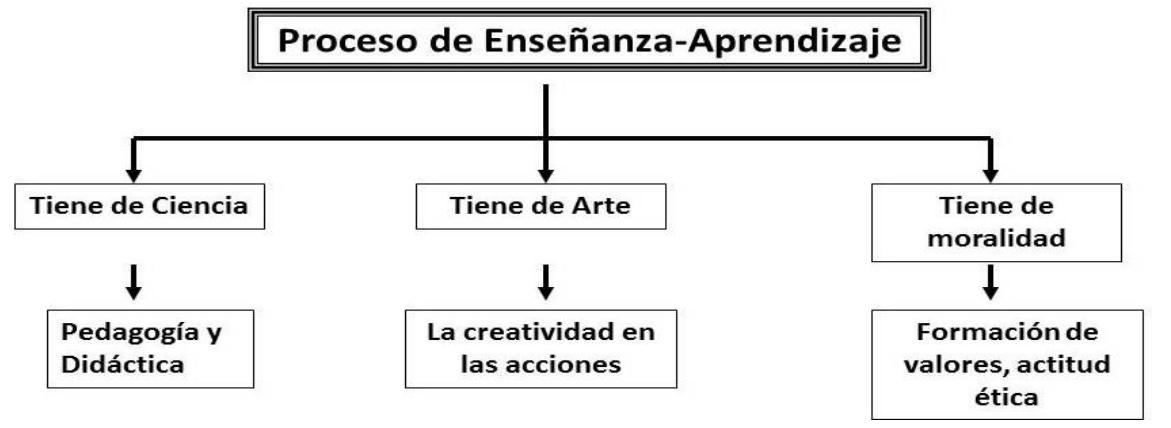

Fig. 1. Diagrama que muestra las características del proceso de enseñanza-aprendizaje

\section{EL MÉTODO CIENTÍFICO, DESCRIPCIÓN Y EVOLUCIÓN HISTÓRICA}

Cuando se habla del Método Científico, se espera generalmente empezar por una definición apropiada; sin embargo cuando se ha intentado hacerlo solo se ha logrado de manera limitada (Rosenblueth, 1971). Así que nos limitaremos en esta sección a comentar solo acerca de la utilización de este método en las ciencias experimentales, particularmente en la física, porque esta rama del conocimiento ocupa un lugar preponderante en la formación de un ingeniero, y porque es en donde mayores éxitos se han obtenido con su uso sistemático.

El Método Científico se basa en lo empírico, en la medición, sujeto a principios específicos de pruebas de razonamiento. Planteado formalmente en el siglo XVII, consiste en la observación sistemática de los fenómenos naturales, medición, experimentación, formulación de hipótesis, análisis y modificación de las hipótesis del comportamiento de estos fenómenos. Se puede afirmar que Francis Bacon (1561-1626) consolidó el método inductivo a través del empirismo, y que fue desarrollado y mejorado entre otros por Pascal (1623-1662), Spinoza (1632-1677), Locke (1632-1704), Malebranche (1638-1715), Newton (16431727), Hume (1711-1776), Kant (1724-1804) y Hegel (1770-1831). 
Sir Francis Bacon en su Novum Organum (Klein, 2016) definió el Método Científico como un proceso con los pasos siguientes: a) observación: aplicar los sentidos a un objeto o a un fenómeno, para estudiarlos tal como se presentan en realidad, puede ser ocasional o causalmente; b) inducción: extraer el principio fundamental de cada observación o experiencia; c) hipótesis: explicación provisional de las observaciones o experiencias y sus posibles causas; d) comprobación de la hipótesis por experimentación; e) demostración o refutación (antítesis) de la hipótesis; f) tesis o teoría científica. La forma esquemática tradicional de presentar al Método Científico es como una sucesión de pasos a seguir, como se muestra en la Figura 2:

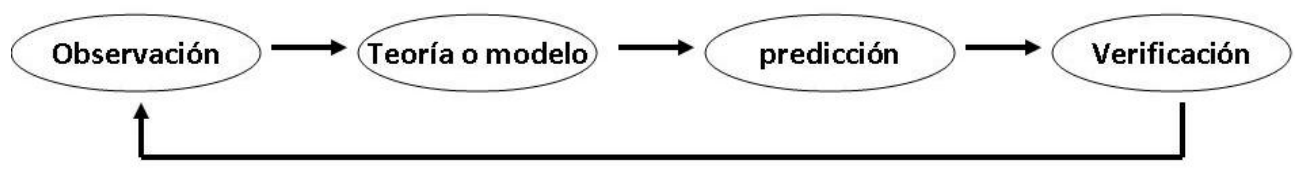

Fig. 2. Diagrama que muestra los pasos principales del Método Científico

Obsérvese que el Método Científico descansa en dos pilares fundamentales: la reproducibilidad, que es la capacidad de repetir un determinado experimento, en cualquier lugar y por cualquier persona, y la comunicación y publicidad de los resultados obtenidos, que debe llevar a su verificación por la comunidad científica; la refutabilidad: que implica que toda proposición científica debe ser susceptible de ser falsada o refutada (falsacionismo), que implica el modus tollendo tollens del método hipotético-deductivo experimental, rechazando las verdades absolutas, puesto que las proposiciones científicas nunca pueden considerarse absolutamente verdaderas, sino a lo sumo "no refutadas" (Lakatos, 1983). El científico usa métodos definitorios, métodos clasificatorios, métodos estadísticos, métodos hipotético-deductivos, procedimientos de medición, entre otros. Referirse al Método Científico es referirse a este conjunto de tácticas empleadas para constituir el conocimiento, sujetas al devenir histórico, y que eventualmente podrían ser otras en el futuro (Holton y Brush, 1988). Basándose en Aróstegui et al. (1978) y Koestler (1959), es posible proponer una breve historia de los antecedentes, surgimiento y desarrollo del Método Científico:

Antigüedad: Sócrates, Platón y Aristóteles, entre otros, propusieron los primeros métodos de razonamiento filosófico, matemático, lógico y técnico.

Época medieval: filósofos, físicos, matemáticos, astrónomos y médicos del mundo islámico, desarrollaron y difundieron la herencia de la filosofía griega (entre otros Alhazen, Al-Biruni y Avicena). Difusión por Europa; Roberto Grosseteste y Roger Bacon (1220-1292), Escuela de Traductores de Toledo.

Edad moderna: nueva filosofía natural. Descartes (1596-1650) en el Discurso del Método define las "reglas del método para dirigir bien la razón y buscar la verdad en las ciencias".

Precursores: Leonardo da Vinci (1452-1519), Copérnico (1473-1543), Kepler (1571-1630) y Galileo Galilei (1564-1642) aplicaban reglas metódicas y sistemáticas. Galileo Galilei: propuso reforzar la idea de separar el conocimiento científico de la autoridad, la tradición y la fe. La filosofía y la ciencia (conocimiento unitario no fraccionado).

Obsérvese también que se forma un ciclo, pero lo más importante de este esquema es que no constituye un dogma que lleve al conocimiento "verdadero", pues cada paso implica una gran cantidad de problemas por resolver, al mismo tiempo implícitamente llevan a la construcción de nuevas teorías que modifiquen, mejoren y substituyan a las existentes en el momento de alguna investigación o estudio, en el denominado "período de ciencia normal" (Blanché, 1980; Kuhn, 1971). Para lograr la mayor utilidad posible de este método, se plantea un esquema ampliado que indica diversas posibilidades de decisión al momento de llegar a resultados acordes con lo observado de la naturaleza, como el diagrama que se muestra en la Figura 3. Pero, sin duda se ha de reconocer que todo conjunto de conocimientos que aspira a obtener la categoría de ciencia, parte del postulado fundamental de que la naturaleza es predecible. Es importante señalar que en el diagrama mostrado en la Figura 3 existen dos puntos importantes de bifurcación, para cuando existe la necesidad de decidir, y dos puntos de postulación que van conectados inmediatamente. Podemos resumir los pasos del diagrama en los siguientes puntos importantes.

Observación: en particular para un sistema físico consiste en tratar de entenderlo e intentar predecir su comportamiento (lo que significa actualmente buscar una manera de medir).

Postulación de un modelo o teoría: consiste en establecer un conjunto de supuestos que necesitan ser verificados, considerándose una teoría como una refinación del modelo.

Predicciones del modelo: su característica principal es que debe predecir el comportamiento del sistema. 
Verificación: en esta acción se somete el modelo del sistema a una revisión que le da consistencia lógica y puede o no asegurar su validez, comparándolo con el comportamiento de la naturaleza que hace el papel de árbitro en cuanto a la validación del modelo. La validez (sí) permite proponer la verificación de resultados, y la no validez (no) obliga a proponer otro modelo o mejorar el anterior.

Reproducción de resultados: resulta crucial mostrar que los resultados del modelo son reproducibles, pues en caso afirmativo (sí) lleva directamente a establecer una nueva teoría que debe contener a la anterior. En caso contrario (no) nos regresa al modelo para mejorarlo o desecharlo al crear uno más satisfactorio.

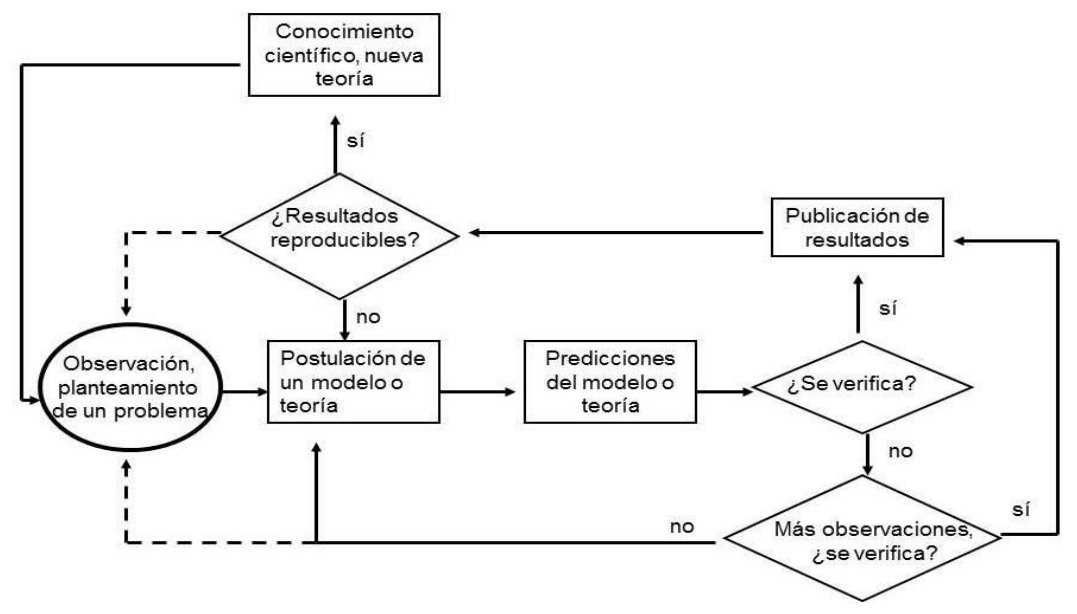

Fig. 3. Diagrama que muestra la manera como se aplica el Método Científico en general

Evidentemente el Método Científico tiene una naturaleza dialéctica, en el sentido de que al momento de crear un modelo válido para un hecho natural, permite crear el posible rival (el contrario), esto permite mejorar dicho modelo. Además muestra que una vez elegido un modelo y ciertas reglas de verificación, no podemos ni debemos transgredir dichas reglas. Se convierte en norma de conducta para cualquiera que desee dedicarse a esta actividad (la ciencia) o sus aplicaciones.

\section{INTERACCIÓN VALORES - MÉTODO CIENTíFICO}

Para hablar apropiadamente de una interacción entre Educación en Valores y Método Científico, es necesario observar que la invención y la innovación generan implícitamente un conjunto de sentimientos, valoraciones, gusto estético, deseo de demostración de las capacidades, desafíos y satisfacción, al vencer estos desafíos y obtener reconocimiento. Éstos son motivos para la creatividad, para el entusiasmo por la profesión y la entrega al trabajo, pero resultaría negativo que entraran en conflicto con los intereses de la sociedad donde el futuro ingeniero se desarrollará. Una posible solución adecuada depende de un sistema de valores presentes en el proceso de enseñanza-aprendizaje, que permitan un desarrollo considerado adecuado del futuro ingeniero, proporcionando equilibrio en el desempeño de su labor. Se hace imprescindible hacer una selección de valores, a partir del modelo de profesional que se desee obtener. Así, en el caso de la ingeniería, se pueden proponer, sin que sean únicos o suficientes, los siguientes valores: eficacia, dignidad, sensibilidad, compromiso social. Se espera que estos valores generen un conjunto de actitudes a través de los valores específicos que se fomenten. Así, como se muestra en la Figura 4, se establece una relación entre las actitudes y los valores fomentados, de manera que exista una jerarquización de dichos valores de modo que se genere un conjunto de actitudes, no necesariamente jerarquizadas. Obsérvese además que en dicha figura se hace notar que existe una retro-alimentación entre valores fomentados y actitudes, de manera que la formación de valores permita el desarrollo de actitudes para enfrentar el aprendizaje y la práctica profesional.

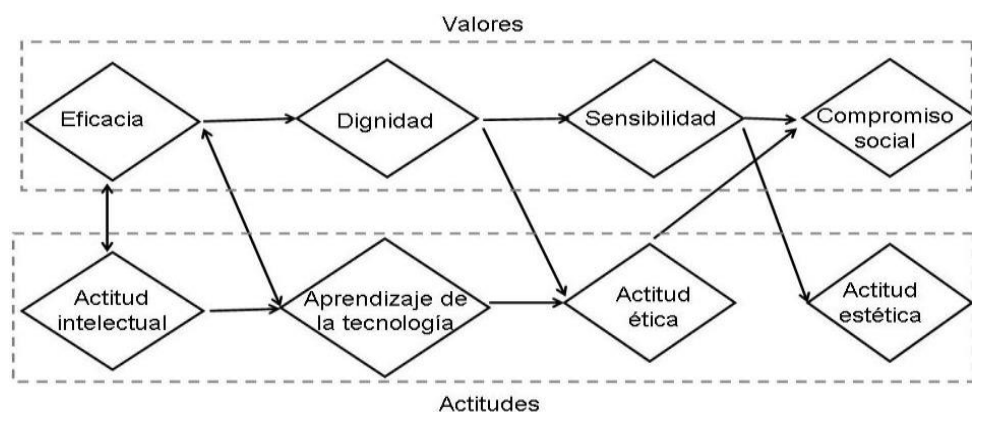

Fig. 4. Relación entre las Dimensiones (actitudes) y Valores seleccionados 
En la práctica del Método Científico también existe una relación entre actitudes y valores, al utilizarlo en la enseñanza de las ciencias duras, como antes se dijo, particularmente en la enseñanza de la física. Un análisis de las actitudes necesarias al momento de utilizar este método nos muestra que existe implícitamente la formación de los valores arriba descritos (Figura 4), como se observa al analizar paso a paso esta correlación. Así, en la Figura 3 es posible establecer tres aspectos importantes que sintetizan la aplicación del Método Científico y se puede construir un esquema como el de la Figura 4, con los aspectos descritos a continuación.

Observación: al plantear un problema se muestra una actitud intelectual de manera que el modelo sea una forma adecuada de representar un hecho o fenómeno natural, se busca ser eficaz al tratar de resolver un problema.

Postular una teoría: para dicho modelo, implica aprender y desarrollar una tecnología, a través de la creación de algoritmos para medir y comparar, buscando la eficacia y con dignidad en la labor desarrollada. Reproducción de resultados: al presentar sólo aquellos resultados que realmente se ha obtenido a partir del modelo, aunque no se ajuste a nuestras predicciones, y que pueden ser reproducibles por cualquier integrante de la corriente de pensamiento

Actitud ética y con sensibilidad, se requiere incluso en caso de tener que regresar al punto inicial, presentando un modelo mejorado que substituya al anterior, esperando que sea simple y estético.

Los cuatro aspectos previamente comentados muestran la necesidad de un compromiso social, al buscar en todo momento mejorar tanto el modelo como la tecnología apropiada para desarrollarlo.

\section{REFLEXIONES SOBRE EL PROBLEMA}

Es conveniente hacer ahora algunas aclaraciones sobre el presente trabajo. Resultaría interesante analizar algunos ejemplos de temas centrales de la Ingeniería o afines a ellos. Pero tal vez tendríamos que precisar a cuál Ingeniería nos estaríamos refiriendo, pues en las universidades de muchos países no existe en especial una carrera de Ingeniería, pero si existen Ingeniería Mecánica, Ingeniería Eléctrica, Ingeniería en Sistemas, Ingeniería en Computación, Ingeniería Química, Ingeniería Ambiental, Ingeniería Hidráulica, etc. (Grech, 2001). Todas ellas tienen un denominador común: el estudio de las matemáticas como herramienta (existe un nivel básico que sirve a todas) y el estudio de la física (también con un número básico de temas que a todas sirve). Introducir una discusión sobre alguna de estas ingenierías, en el presente trabajo, excedería los objetivos que se han planteado en forma general, pues la exposición se centra en mostrar las similitudes entre los objetivos intrínsecos del Método Científico en las ciencias duras necesarias en las ingenierías, y los objetivos explícitos de la teoría de la Educación en Valores. Esto es, el planteamiento es de carácter comparativo y no específico de una carrera o una asignatura.

La teoría de la Educación en Valores parte de la idea de que debe ser posible guiar al estudiante, en su propia búsqueda de valores, a través del aprendizaje de cualquier rama del conocimiento que se enseñe en el nivel universitario, sin importar si es una ciencia dura o una disciplina que no llegase a considerarse ciencia, pero en ningún estudio, por más serio que sea, existe o se sugiere una metodología que muestre la manera de hacerlo. Una alternativa puede ser el hacer énfasis en el Método Científico como una metodología para entender la evolución del conocimiento. Es conveniente recordar que para llamar ciencia a una disciplina o conjunto de conocimientos, es necesario que sea susceptible de un desarrollo mediante el uso sistemático del Método Científico. En caso contrario, si no se ajusta a ese desarrollo, sólo será una disciplina más, útil pero no una ciencia, y recordemos que esto es lo que hace esencialmente dialécticas a las ciencias. La aplicación de la teoría de Educación en Valores también será posible en estas disciplinas, considerándose apropiada para obtener ingenieros más comprometidos con su profesión y su entorno.

Además, cabe señalar que existen muchos estudios sobre el problema de la Educación en Valores para futuros ingenieros, en los que se abordan diversos tópicos del problema: caracterización de los "atributos" que se espera debe tener un profesional en ingeniería (Covarrubias,1998), valoración de las actitudes relacionadas con la ciencia (Manassero y Vázquez, 2001), análisis de las teorías de la enseñanza (Loo, et al, 2003), la educación en valores y su aplicación en el proceso de enseñanza-aprendizaje (del Valle, 2004; Lozano et al, 2003; Parra, 2003; Valiente, 2004). Recientemente han aparecido diversos estudios que abonan la discusión sobre la teoría de la Educación en Valores y el problema de su inclusión en el proceso de enseñanza-aprendizaje en carreras de ingeniería (Covarrubias-Villa y Cruz-Navarro, 2011; González y Rasilla, 2011; Osuna y Luna, 2008). De todos estos trabajos se puede inferir que el problema no es simple y que tiene muchas facetas.

En cualquiera de las especialidades de la ingeniería, como ya se ha comentado, es esencial el aprendizaje de las ciencias naturales. Una aspiración central en este contexto es la aspiración de saber y entender, pero 
para los futuros ingenieros este aprendizaje es útil pero de forma diferente que para un futuro científico. El estudiante de ciencias está motivado por la curiosidad y en cambio el futuro ingeniero busca atacar los problemas de la vida real y resolverlo, sin importar si son o no comprendidos los principios básicos de la ciencia particular que haya utilizado. Se espera que el ingeniero sea un innovador, para lo cual se requiere que tenga las características siguientes: receptivo de las necesidades humanas, capaz de saber buscar recursos materiales y humanos necesarios para resolver un problema, capaz de desarrollar un proceso de producción hasta llegar a una etapa de difusión masiva. Aquí es donde aparece la necesidad de una Educación en Valores, que se puede lograr a través de la discusión de la manera de como se ha llegado al grado de conocimientos actual. Esto es, a través de la exposición del desarrollo histórico de las ciencias, mostrando como ha influido el Método Científico en dicho desarrollo.

\section{CONCLUSIONES}

Del estudio presentado, se pueden extraer las siguientes conclusiones principales:

En el desarrollo del proceso de enseñanza-aprendizaje para las ciencias duras, es posible aprovechar la utilización del Método Científico, para incluir en la enseñanza la apropiación de valores, por parte del estudiante, que moldeen los rasgos o características de la personalidad del futuro ingeniero, en aras de una actitud de servicio hacia la sociedad que lo ha entrenado, no solo para resolver problemas prácticos, también para tener una actitud digna ante los problemas y retos que se le presenten en su vida profesional.

La Educación en Valores, como teoría pedagógica, puede ser incluida en el momento en que el Método Científico es puesto en práctica como una forma de exposición de la manera en que el desarrollo de las teorías científicas han ido apareciendo, el estudiante puede verse reflejado como el futuro agente de cambio y desarrollo de la sociedad en que vive.

Al analizar detenidamente los aspectos principales de la Educación en valores y el Método Científico, se hallan coincidencias entre los objetivos de una y otro, formándose una simbiosis, que puede ser aprovechada por los profesores, durante el proceso de enseñanza-aprendizaje, para beneficio del futuro profesionista en particular, y la sociedad que lo prepara para la vida en general. La teoría de la Educación en Valores y el Método Científico aparecen como una sistematización metodológica para infundir en los futuros ingenieros actitudes y valores que los hagan útiles a la sociedad de la que reciben una profesión.

\section{AGRADECIMIENTOS}

Los autores agradecen el apoyo de sus respectivas Instituciones en el desarrollo del presente trabajo. Especialmente agradecen los amables comentarios de los revisores que permitieron mejorar el trabajo.

\section{REFERENCIAS}

Arana, M.H. y M. del C. Armenteros, Formación Sociohumanista en las Carreras de Ciencias Técnicas en Cuba, Contactos, III (23), 26-29 (1996)

Arana, M.H. y N. Batista, La Educación en Valores: una Propuesta Pedagógica para la Formación Profesional, ISPAJAE, Ministerio de Educación Superior (Cuba), La Habana, Cuba (1997)

Aróstegui, J.M., A. Bustamante y otros 10 autores, Metodología del Conocimiento Científico, 11-217, Editorial de Ciencias Sociales, Instituto Cubano del Libro, La Habana, Cuba (1978)

Baños-Martínez J.J. y M.I. Arganis-Juarez, The Formation in Moral Values in High School Education by Means of the Transversal Axis and the Integrated Curriculum, Procedia-Social and Behavioral Sciences, 106, 2807-2816 (2013)

Barba, B., Educación y Valores: una Búsqueda para Reconstruir la Convivencia, Revista Mexicana de Investigación Educativa, 10 (24), 9-14 (2005)

Batista, N. y D. Reyes, La Integralidad en la Formación del Egresado: un Imperativo Universitario, Contactos, III (22), $37-$ 43 (1997)

Berkowitz, M. W., What Works in Values Education, International Journal of Educational Research, 50, 153-158 (2011)

Blanché, R., El Método Experimental y la Filosofía de la Ciencia, traducción del francés por A. Ezcurdia, Fondo de Cultura Económica, México, México (1980)

Carr, D., Values, Virtues and Professional Development in Education and Teaching, International Journal of Educational Research, 50, 171-176 (2011)

Castiglione, C., A. Rampullo y O. Licciardello, High School Students' Value System, Procedia-Social and Behavioral Sciences, 141, $1330-1334(2014)$

Córdova, J. L. y A. Rojo, Actitudes Científicas en el Aprendizaje de Ciencias, Contactos, II (7), 52-61 (1985) 
Covarrubias, J. M., Tres Elementos Sobre la Formación de Ingenieros, Ingenierías, 1 (1), 5-9 (1998)

Covarrubias-Villa, F. y M. G. Cruz-Navarro, El Problema de la Racionalidad en la Didáctica de la Ciencia, Formación Universitaria, 4 (6), 49-60 (2011)

Del Valle, A. I. y E. Usategui, Los Valores en la Enseñanza de la Ingeniería, Revista de Enseñanza Universitaria, 29, 5167 (2007)

Einstein, A., On the Method of Theoretical Physics, Philosophy of Science, 1 (2), 163-169 (1934)

Garcell, M., Teorías y Estrategias para la Formación de Valores en la Educación, Cuadernos de Educación y Desarrollo, 2 (17), (2010)

González, L. M. y M. Rasilla, Una Estrategia para el Aprendizaje de la Cultura Científica, Formación Universitaria, 4 (2) 15-26 (2011)

Grech, P., Introducción a la Ingeniería, 9-34, Pearson Educación, Colombia (2001)

Halstead, J.M. y M.J. Taylor, The Developments of Values Through the Life of School, en: The Development of Values, Attitude and Personal Qualities, National Foundation of Educational Research, 17-33, Berkshire, U.K. (2000)

Holton, G. y S.G. Brush, Introduction to Concepts and Theories in Physical Sciences, Second Edition. Traducción de J. Aguilar: Introducción a los Conceptos y Teorías de las Ciencias Físicas, 251-328, Reverte, Madrid, España (1988)

Hoyos, G., M. Martínez y otros 9 autores, La Educación en Valores en Iberoamérica, Colección: Papeles Iberoamericanos, Organización de Estados Iberoamericanos para la Educación la Ciencia y la Cultura (OEI) (2001)

Klein, J., F. Bacon, en The Stanford Encyclopedia of Philosophy (Winter 2016). Stanford University Press, Stanford, USA (2016)

Koestler, A., The Sleepwalkers, a History of Man's Changing Vision of the Universe, Hutchinson \& Co., Ltd., London, U.K. (1959)

Kuhn, T. S., The Structure of Scientific Revolutions, Chicago University Press, Chicago, USA (1962)

Lakatos, I., The Methodology of Scientific Research Programs, Cambridge University Press, London, U.K. (1978)

Loo, I., A. Olmos y A. Granados, Teorías Implícitas Predominantes en Docentes de Cinco Carreras Profesionales. Rev. Enf. IMSS 11 (2), 63-69 (2003)

Lovat, T., N. Clement, K. Dally y R. Toomey, The Impact of Values education on School Ambience and Academic Diligence, International Journal of Educational Research, 50, 166-170 (2011)

Lozano, F., A. Boni, J. C. Siurana y C. Calabuig, La Enseñanza de Valores Estéticos en las Carreras Científico-Técnicas Monografías Virtuales (U. de Valencia) 3, 1-7 (2003)

Manassero, M.A. y A.A. Vázquez, Instrumentos y Métodos para la Evaluación de las Actitudes Relacionadas con la Ciencia, la Tecnología y la Sociedad, Enseñanza de las Ciencias, 20 (1), 15-24 (2001)

Osuna, C., E. Luna, Características de Ser un Buen Profesional de Ingeniería en la Universidad Autónoma de Baja California, México, Formación Universitaria, 1 (1), 29-36 (2008)

Parra, J. M., La Educación en Valores y su Práctica en el Aula, Tendencias Pedagógicas, 8, 69-88 (2003)

Poincaré, H., Filosofía de la Ciencia (colección de ensayos), 39-100, CONACYT, México, México (1981)

Popper, K., The Logic of Scientific Discovery; Corroboration, or How a Theory Stands up to Tests, 248-282, Taylor \& Francis e-Library, London U.K. (2005)

Rosenblueth, A., El método Científico, CIEA-IPN- CONACYT, México (1971)

Şahinkayasi, Y., y Ö. Kelleci, Elementary School Teachers' Views on Values Education, doi: 10.1016/j.sbspro.2013.09.162, Procedia-Social and Behavioral Sciences, 93, 116-120 (2013)

Schmelkes, S., La Formación de Valores en la Educación, Estudios (Filosofía-Historia-Letras). ITAM, México, México (1996)

Sheldrake, R., T. Mujtaba y M. J. Reiss, Science Teaching and Students' Attitudes and Aspirations: The Importance of Conveying the Applications and Relevance of Science, International Journal of Educational Research, 85, 167-183 (2017)

Simmons, C., Education, Culture and Values, Volume II, 58-74, Taylor \& Francis e-Library, London, U. K. (2005)

Thornberg, R., The Lack of Professional Knowledge in Values Education, doi:10.1016/j.tate.2008.04.004, Teaching and Teacher Education: An International Journal of Research and Studies, 7 (24), 1791-1798 (2008)

Valiente, A., Los Valores y la Ingeniería Química, Educación Química, 16 (2), 320-325 (2003) 